\title{
Broadband microwave metamaterial absorber with lumped resistor loading
}

\author{
Ke Chen ${ }^{*}$, Xinyao Luo, Guowen Ding, Junming Zhao, Yijun Feng, and Tian Jiang \\ School of Electronic Science and Engineering, Nanjing University, Nanjing 210093, PR China
}

Received: 30 September 2018 / Accepted: 14 December 2018

\begin{abstract}
Narrow absorption bandwidth has been a fundamental drawback hindering many metamaterial absorbers for practical applications. In this paper, by loading lumped resistors, we have successfully designed a microwave metamaterial absorber with multioctave wide absorption bandwidth covering the entire $\mathrm{X}$ - and $\mathrm{Ku}$ bands, while keeping the thickness of the absorber less than $1 / 10$ of the working wavelength. The polarizationinsensitive absorber shows a good angular stability for both transverse electric (TE) and transverse magnetic (TM) incidences. Prototype has been fabricated and measured to validate the design principle and the simulated results, and good agreements are observed between simulated and measured results. The proposed metamaterial absorber offers an efficient way to realize broadband microwave absorption with stable angular performance, which may find potential uses in many applications, for example, electromagnetic compatibility.
\end{abstract}

Keywords: Metamaterial absorber / broadband / microwave / lumped resistor

\section{Introduction}

The electromagnetic (EM) wave absorbers, which are viewed as EM blackbodies that can efficiently inhibit reflection and transmission of EM radiation, have attracted much attention due to their practical uses in many real-world applications such as electromagnetic compatibility (EMC), stealth technique, etc. [1]. Recently, metamaterials composed of artificially engineered subwavelength inclusions have shown great abilities in designing diverse absorber designs, due to their designable features of EM properties that are not available in natural occurring materials $[2,3]$. Therefore, unremitting efforts have been made to enhance the absorption performances with factors such as polarization-insensitive [4], switchable or active control [5,6], ultrathin thickness [7], and wide incident angle [8] across the whole spectrum ranging from microwave to optics. However, narrow working bandwidth still lays remarkable limitation, thus hindering their further applications.

In order to achieve excellent absorption and broad working bandwidth simultaneously, several methods, including employing tapered or multilayered structures $[9,10]$ and multiresonant structures $[11,12]$, have been implemented. Despite the improvement of the absorption bandwidth, some problems have been introduced by these methods, e.g., increase of total thickness and difficulty in

\footnotetext{
* e-mail: ke.chen@nju.edu.cn
}

assembly, as well as possibility of resulting discrete absorption bands. Designing absorbing structures with suitable lossy components have also been demonstrated as an efficient way to realize broad operation band. One of the most well-known structures is the Salisbury screen absorber [13], with a thin homogeneous resistive sheet suspended above a ground plane by a distance of a quarter of a wavelength. By replacing the ground plane with judiciously designed metasurface, the bandwidth of the Salisbury screen can further be significantly improved due to the excitation of multiple metasurface Salisbury screen resonances [14,15]. Besides, loading with other types of lossy components such as magnetic medium [16,17], resistive sheets [18,19], lumped elements [20-23], and lossy liquid water [24,25] can be good candidates for potentially broadening the absorption bandwidth, but most of them are limited by the angular stability, which may further hinder their practical uses.

In this paper, we design a planar polarization-insensitive, angular stable, and thin-thickness microwave metamaterial absorber loaded with lumped chip resistors, which are capable of absorbing EM energy over a multi-octave broad frequency band covering the entire X-band $(8-12 \mathrm{GHz})$ and $\mathrm{Ku}-$ band $(12-18 \mathrm{GHz})$. The proposed metamaterial absorber has a comparable thin-thickness less than $0.067 \lambda$ (with respect to the lowest frequency of working band). Especially, by loading a set of metallic via holes, the magnetic resonances of the structure have been enhanced throughout the working band, resulting in a good angular stability for incident angle up to about $\pm 60^{\circ}$ for 


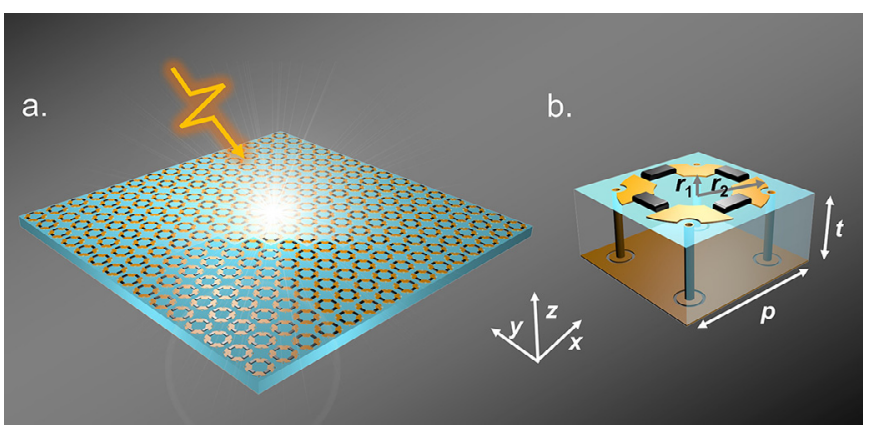

Fig. 1. (a) Schematic of the proposed metamaterial absorber, which can efficiently absorb the incident wave in a broad frequency band. (b) Details of the unit cell configuration. Four metallic via holes are embedded into the dielectric substrate and connected to the metallic circular patches that are isolated from the ground with a gap width of $0.1 \mathrm{~mm}$. The via hole has a radius of $0.15 \mathrm{~mm}$, while the patch has a radius of $0.4 \mathrm{~mm}$. Four semicircular corners are cut from the metallic ring structure on the top layer.

both transverse electric (TE) and transverse magnetic (TM) waves. Finally, experiments have been carried out to validate the design principle and the simulated results, and good agreements are observed between simulated and measured results.

\section{Design considerations and simulation}

To realize efficient wave absorption, the metamaterial structures are often designed to resonate at certain frequencies, and when these structures are shined by the incidence, field enhancement occurs in structures, which then leads to energy dissipations caused by the lossy components, for example, lossy dielectric substrates. Although the dielectric loss can result in a perfect absorption [26], it is often limited to relatively narrow bandwidth due to the sharp structural resonance. Therefore, we usually increase the lossy component to reduce the $Q$-value of the resonances by using, for example, resistive film, resistor, etc., and to broaden the absorption bandwidth. Figure 1a shows the schematic of the proposed metamaterial absorber. When a plane wave propagating along the $z$-direction, either with $x$ - or $y$-polarization, incidents on the metamaterial absorber, the wave energy will be efficiently dissipated by the proposed absorber in a broad frequency band. Figure 1b shows the details of the unit cell configuration. The unit cell consists of three layers, where the lossy dielectric spacer is sandwiched between patterned top metallic layer and bottom ground plane. The dielectric substrate has a thickness of $2.5 \mathrm{~mm}$, with relative permittivity of 4.4 and loss tangent of about 0.025 . The metallic material used herein is the copper with conductivity of $5.8 \times 10^{7} \mathrm{~S} / \mathrm{m}$ and a thickness of $0.018 \mathrm{~mm}$. The employed top layer is shaped in a circular ring with four split gaps in two orthogonal directions cut away and replaced by four chip resistors. These resistors could ensure an efficient energy dissipation when the surface current, induced by the incident illumination, flows around the patterned structures. Four metallic via holes are perforated in the structure to enhance the magnetic resonances. When the structure is exposed to an incident wave with magnetic field travels across via holes, comparable anti-parallel currents can be induced and flow along the neighboring metallic via holes, which in turn enhance the energy absorption. Due to the rotational symmetry and mirror symmetry in the metamaterial structure, the proposed absorber presents polarization-insensitive property to the incident EM wave.

The broadband absorption performance of the metamaterial absorber is first evaluated by the full-wave simulation using commercial software. Since the absorber is composed of periodically arranged elements, we can only investigate the performance of the constituent element instead of calculating overall performance of the whole metamaterial. In the full wave simulation setup, we apply perfect electric conductor (PEC) boundary condition along one direction, while perfect magnetic conductor (PMC) boundary condition along the orthogonal direction. These boundary conditions are used to mimic the periodic arrangements. In addition, free space boundary conditions are applied along the $z$-direction. It should be noted that these boundary conditions actually form a waveguide terminated by the ground plane of the structure, and we can calculate the absorption performance from the $S$ parameters. Since there are four circular slots existing on the ground plane, we should include the transmission coefficient in the absorption calculation. Therefore, this calculation can be written as $A(f)=1-T(f)-R(f)$, where $A(f), T(f)$, and $R(f)$ are the frequency-dependent absorption, transmission, and reflection, respectively. The final optimized geometrical parameters of the proposed metamaterial absorber are $t=2.5 \mathrm{~mm}, p=5.6 \mathrm{~mm}, r_{1}=1.3 \mathrm{~mm}$, $r_{2}=2.5 \mathrm{~mm}$, with the chip resistor set as $110 \Omega$.

The full-wave simulated results are shown in Figure 2a, where the absorption bandwidth defined by reflection coefficient lower than $-10 \mathrm{~dB}$ ranges from about 7.95 to 18.5 GHz. The transmission of the proposal uniformly keeps lower than $-35 \mathrm{~dB}$ across the entire working band, which means that negligible EM wave energy can pass through the unit cell structure, indicating that we could derive the absorption only by taking consideration of the reflection wave. The frequency-dependent absorption, transmission, and reflection curves under normal illumination of the incidence are shown in Figure 2b. The transmitted EM wave energy is always less than 0.001, indicating that less than $0.1 \%$ of the total incident power can penetrate through the metamaterial absorber. At the same time, the absorption band can cover the entire $\mathrm{X}$ - and $\mathrm{Ku}$-bands, as indicated by the dotted line. As a comparison, the absorber without via holes loading only has a single absorption peak in low frequencies, while the absorption rate rapidly decreases as the frequency increases, leading to a much narrowed absorption bandwidth. Clearly, we can realize efficient absorption for both two incident wave polarizations ( $x$ - and $y$-polarization) in a very broadband frequency band within a subwavelength thickness.

We then perform parametric analyses by varying the parameters of the proposed metamaterial absorber to investigate their effects on the absorption performance. 
a.

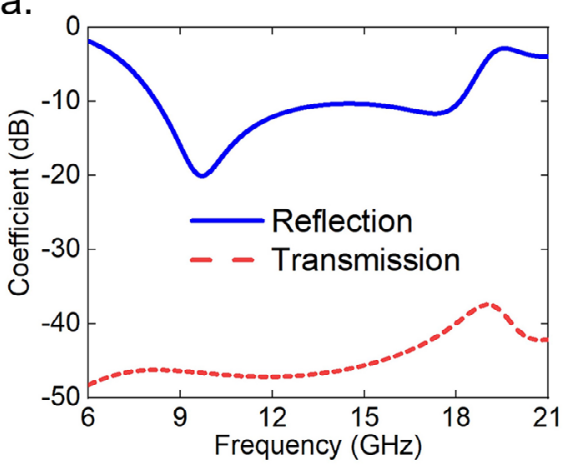

b.

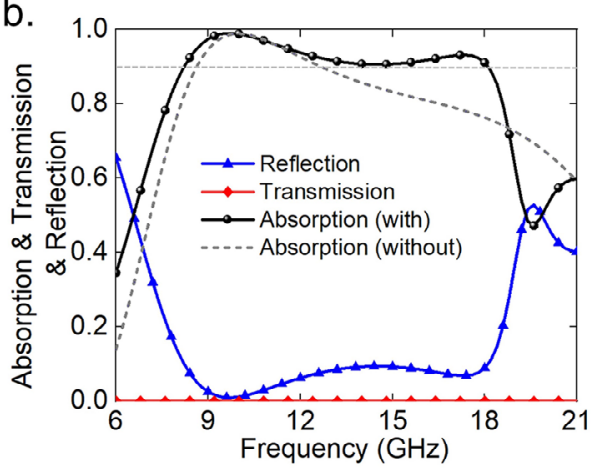

Fig. 2. (a) Simulated reflection and transmission coefficient of the proposed metamaterial absorber. (b) Simulated frequencydependent absorption, transmission, and reflection of the metamaterial absorber. Dotted curve shows the absorption of the absorber without via holes.

a.
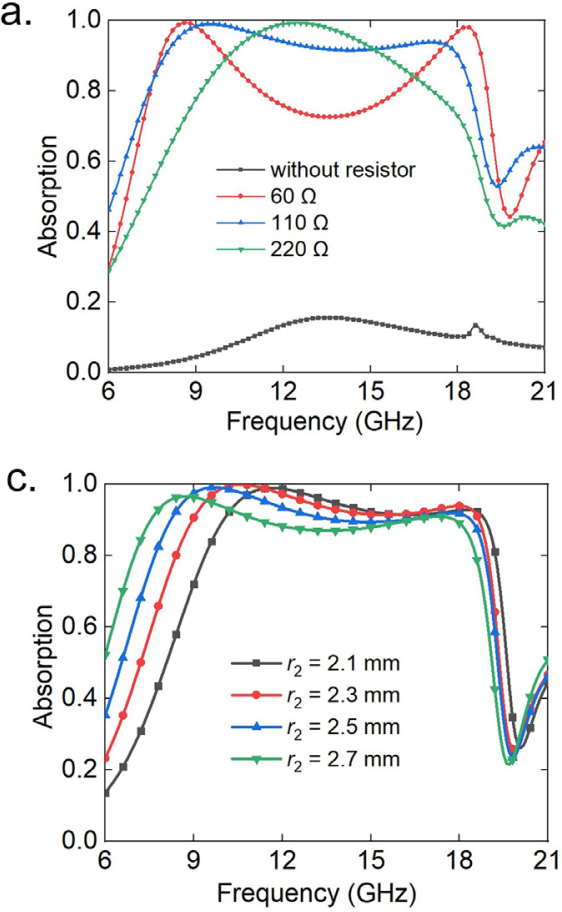

b.

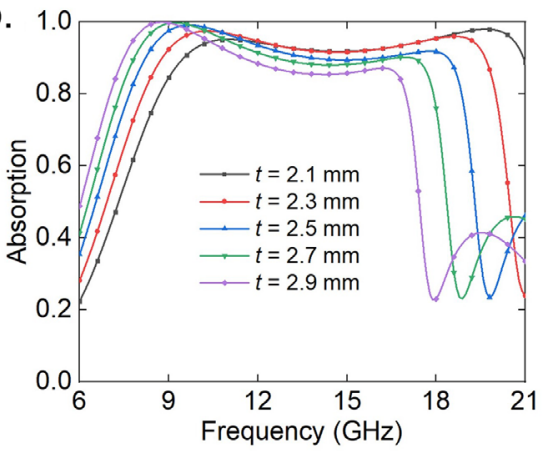

d.

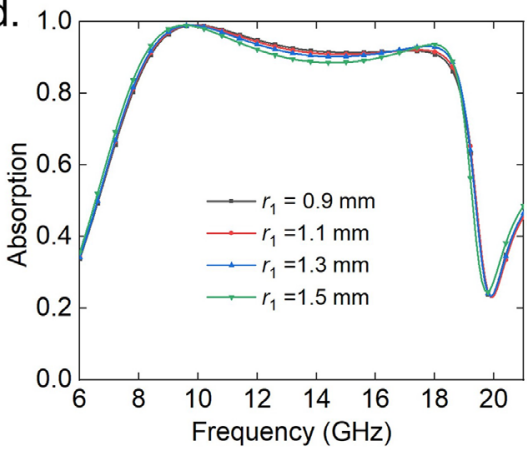

Fig. 3. Parameter study of the proposed metamaterial absorber. The frequency-dependent absorption performance of the absorber as a function of (a) the numerical value of the chip resistors, (b) the dielectric substrate thickness $t$, (c) the outer radius $r_{2}$ of the split circular ring on the top layer, and (d) the inner radius $r_{1}$ of the split circular ring on the top layer.

Four main parameters are studied herein: the radius $r_{1}$ and $r_{2}$ of the metallic ring pattern on the top layer, the thickness $t$ of the dielectric substrate, and the numerical value of the chip resistors. We vary only one parameter in the parametric study and keep other parameters unchanged as the optimized values. The simulated absorption performance of the proposed absorber as a function of the resistance is shown in Figure $3 \mathrm{a}$. When the resistance is increased to $220 \Omega$, the two absorption peaks are merged together, resulting in a reduced bandwidth. On the contrary, when the resistance is reduced to $60 \Omega$, more efficient absorption can be observed at two resonant frequencies, but the continuously broad absorption band is divided into two discrete bands, with the middle band rapidly reduced to a level of about $70 \%$ absorption. It concludes that the embedded resistance values should be properly chosen to obtain efficient wave absorption, and the resistance has a small influence on the resonant frequency when the values are around $110 \Omega$. As depicted by the black curve, when the metamaterial absorber is 
a.

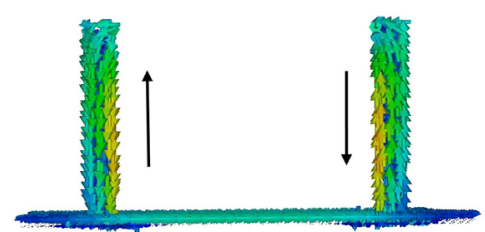

b.

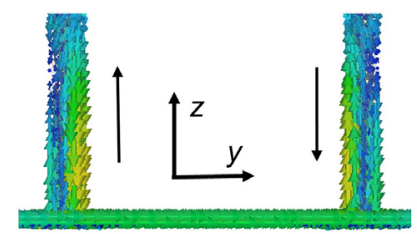

C.

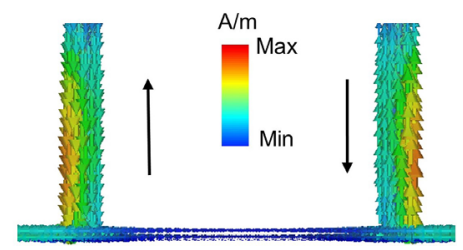

Fig. 4. Surface current distributions on the metallic via holes under the illumination of a $y$-polarized EM wave at the frequency of (a) $10 \mathrm{GHz}$, (b) $14 \mathrm{GHz}$, and (c) $18 \mathrm{GHz}$.

designed without any chip resistor loaded to connect the splits of the rings, the absorption is very weak across 8-18 GH. Therefore, we can conjecture that the major contribution to the wideband absorption is attributed to the energy consumed by chip resistors.

Figure $3 \mathrm{~b}$ shows the absorption rate as a function of the substrate thickness. It can be observed that the thickness has a great influence on the absorption band. The absorption band moves to lower frequency region with the increase of the substrate thickness, while the absorption efficiency can be roughly kept immune from the shift of the working band. Actually, the relative bandwidth and the relative thickness, with respect to the working band, are kept nearly unchanged during this parametric change. One may envision that, by suitably designing other parameters, the working frequencies can be freely designable and a scalable design of this absorbing structure could be fully realized. Figure $3 \mathrm{c}$ shows the parameter scanning of the outer radius of the circular pattern on the first layer. As the parameter $r_{2}$ increases, the first resonant peak shifts toward lower frequency region, resulting in a broadened bandwidth but accompanied with reduced absorption efficiency, especially in low-frequency region. However, the varying parameter influences the absorption efficiency around the second resonant peak, which does not have much influence on the resonant frequency. Besides, one can do a trade-off between bandwidth consideration and absorption efficiency by flexibly altering this parameter. Finally, we also execute the influence of the inner radius of the circular pattern on the overall performance, as shown in Figure 3d. This parametric change only has little change on the absorption performance of the proposed metamaterial absorber.

As we mentioned earlier, the metallic via holes are used to enhance the magnetic resonances and therefore to improve the absorption performance in a broadband. Here, we also investigate the current distributions on the via holes to give an insightful view of how these via holes couple with the incidence. Figure 4 shows the broadband behavior of the surface currents flowing on the metallic via holes under the normal wave excitation. Clearly, antiparallel currents can be observed between neighboring via holes across the entire working bandwidth, indicating magnetic resonances occur. At low frequencies of 10-14 GHz, strong surface currents exist on the inner sides of two neighboring via holes within a unit cell, while at a high frequency of $18 \mathrm{GHz}$, the strong currents tend to appear on other sides of the via holes. The via holes can also benefit the miniaturization of the unit cell, which could further offer stable angular performances. Four circular patches are used on the bottom structure for enabling the metallic via holes to be physically isolated from the metallic ground plane, avoiding direct current flow between these two components. Similarly, the via holes are also physically isolated from the top metallic patterns.

The working mechanism of the proposed structure can be qualitatively analyzed by the transmission line theory [19]. The equivalent circuit model of the top layer can be viewed as a serial RLC resonant circuit, which will show a capacitive response at low frequencies while gradually changing to inductive response as the frequency increases, due to the foster theorem [27]. On the other hand, the grounded dielectric substrate can be viewed as a fraction of transmission line, with the propagation length about onefourth of a wavelength compared to the center working frequency [28]. Such a transmission line will show inductive response in low-frequency band, while capacitive response in higher frequency band. This property is actually opposite to that of the RLC resonance of the first metallic pattern layer. These two opposite responses arisen from the shorten dielectric substrate and the structure on the top layer, when carefully designed, can result in two absorption resonances with one in low frequency and the other in high frequency [19]. In addition, if the resistive components on the first layer are elaborately optimized, the input impedance of structure can be adjusted nearly equal to that of free space, further resulting in a continuous wideband absorption.

Angular stability or angular performance is a very important criterion to evaluate the metamaterial absorber. The above discussions are only related to the case of normal incident wave. Here, we have also investigated the absorption performance of the proposal for different incident angles. The full-wave simulated results for oblique incidence are shown in Figure 5. Both TM and TE modes are considered. The TM wave is defined as the magnetic field perpendicular to the incident plane while the electric field is parallel to it, and the TE wave is defined as the electric field perpendicular to the incident plane while the magnetic field is parallel to it. In the simulation, unit cell boundary condition is applied to the unit cell of the proposal both in $x$ - and $y$-directions. Figure 5a depicts the angular-dependent absorption for TM incidence. As the incident angle increases, the absorption efficiency has gradually increased but the bandwidth reduces. For TM case, the magnetic field always travels across the loops 
a.

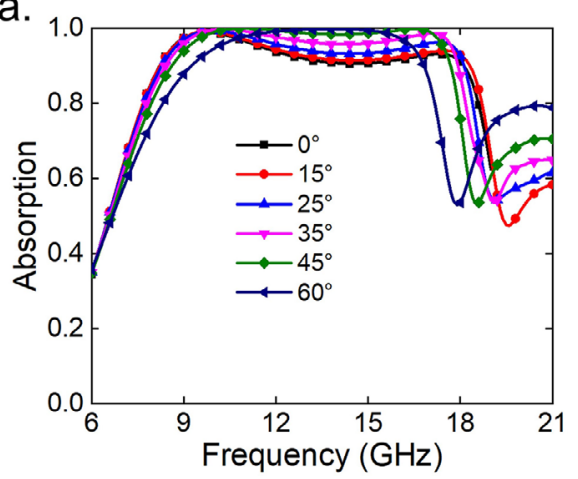

b.

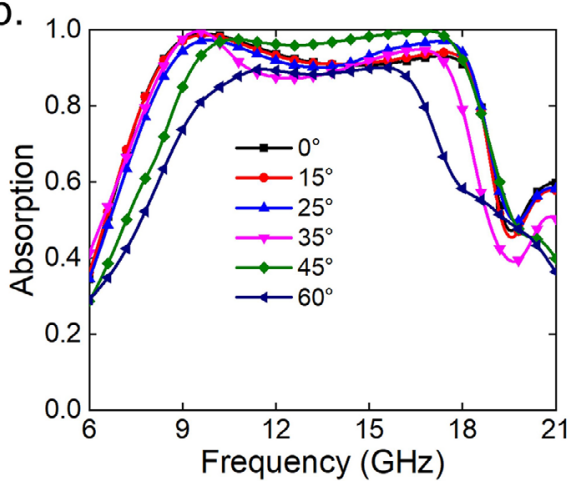

Fig. 5. Simulated angular performance of the proposed metamaterial absorber under the illumination of (a) TM- (b) TE-polarized incidence.
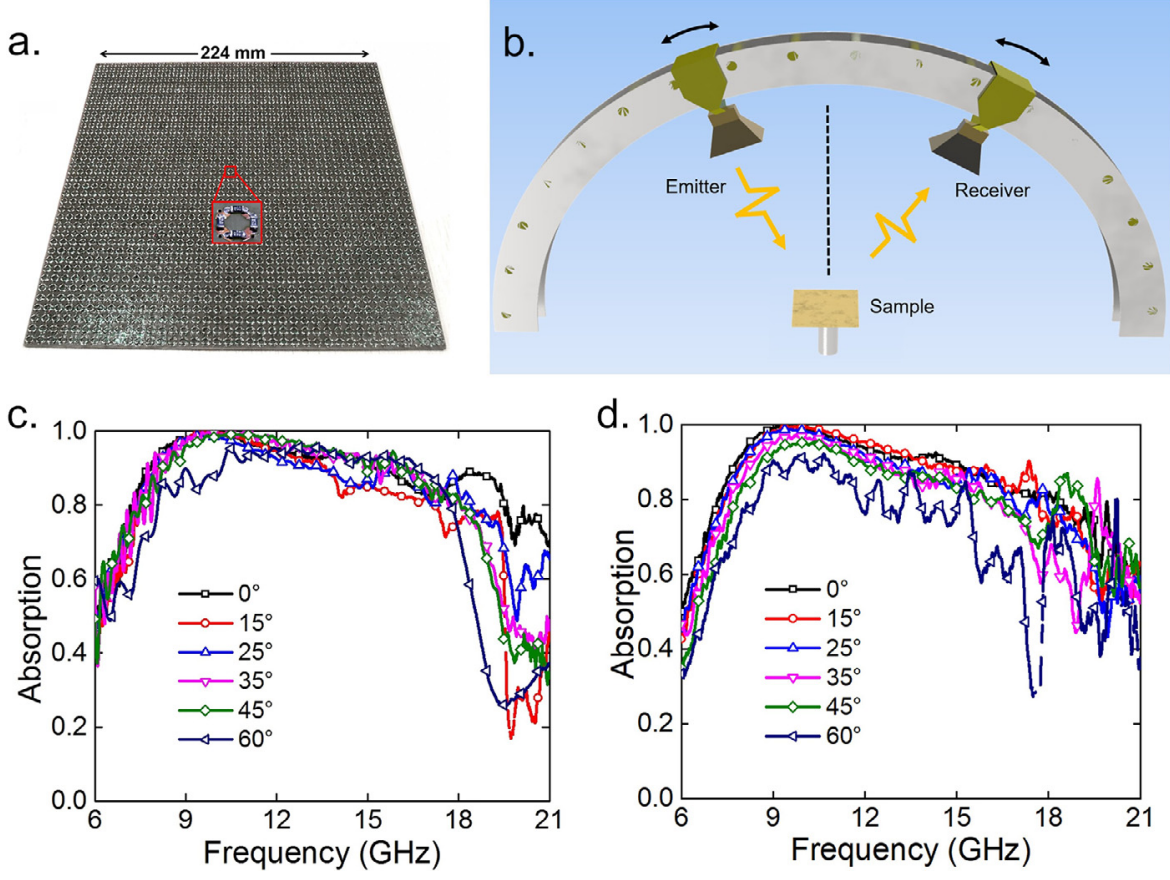

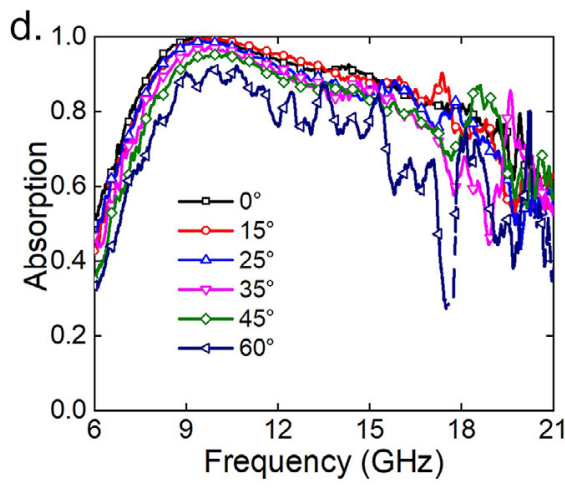

Fig. 6. (a) Photograph of the fabricated metamaterial absorber. The inset shows the enlarged view of a single unit cell. (b) Schematic for the experimental setup for reflection measurement. (c) Measured angular performance of absorber under the illumination of TM-polarized incidence. (d) Measured angular performance of absorber under the illumination of TM-polarized incidence.

composed by the via holes, leading to an enhanced magnetic resonance nearly immune from the incident angle changing. For incident angle up to about $60^{\circ}$, the proposed absorber can still have an absorption bandwidth defined by 0.9 , ranging from 9.3 to $17 \mathrm{GHz}$. Figure $5 \mathrm{~b}$ depicts the angular-dependent absorption for TE incidence. Clearly, the absorption performance is well preserved for incident angle up to about $45^{\circ}$, while it is rapidly reduced as the incident angle further increases to $60^{\circ}$. This is due to fact that the resonant structure has a comparable dependence on the magnetic coupling with the incident wave. Under the illumination of TE-polarized wave, the component of magnetic field travelling across the metamaterial absorber becomes less, especially at large incident angles. The differences between TE- and
TM-polarized cases are mainly attributed to the structural dispersion within finite element size, as well as the intrinsic wave impedance different for two polarized incidences [15]. The above results verify that the proposed metamaterial absorber has a relatively good angular performance, especially for TM-polarized incidence.

\section{Experimental verification}

To verify the design principle and the simulated results, we have fabricated a prototype of the proposed metamaterial absorber through standard printed circuit board (PCB) technique. The fabricated sample has dimensions of $224 \times 224 \mathrm{~mm}$ containing 1600 unit cells, and chip resistors 
Table 1. Comparison with other works.

\begin{tabular}{llll}
\hline Absorber & Bandwidth (relative) & Thickness & Polarization \\
\hline Ref. [9] & $0.8-1.28 \mathrm{THz}(46.2 \%)$ & $0.069 \lambda$ & Single \\
Ref. [20] & $7.7-21.7 \mathrm{GHz}(95.2 \%)$ & $0.175 \lambda$ & Single \\
Ref. [22] & $1-2.05 \mathrm{GHz}(68.9 \%)$ & $0.13 \lambda$ & Both \\
This work & $7.95-18.5 \mathrm{GHz}(79.8 \%)$ & $0.066 \lambda$ & Both \\
\hline
\end{tabular}

are then soldered onto the sample, as shown in Figure 6a. The experimental measurements are carried out in a standard microwave chamber, with experimental setup illustrated in Figure 6b. Two broadband linear-polarized horn antennas are used as the emitter and the receiver, which are then connected to the two ports of a vector network analyzer, respectively. The horn antenna can be reconfigurable to $x$ - or $y$-polarization by its self-rotation with angles of $0^{\circ}$ and $90^{\circ}$. The antennas can be freely moved to different angles along a circular trajectory to mimic the oblique incident cases. The antennas are placed away from the fabricated sample with enough distance to satisfy the far-field condition, thus mimicking the plane wave illumination. Absorbing materials are used in the chamber to get rid of unwanted EM waves bounced from the ground or surroundings. The measured results for TM- and TE-polarized incidence are shown in Figures $6 \mathrm{c}$ and $6 \mathrm{~d}$, respectively. Good absorption performances covering the entire frequency band from 8 to $18 \mathrm{GHz}$ are observed for both polarizations at normal incident case. The results of TM cases have a good angular stability, where the absorption performances are not influenced much for the incident angle up to about $60^{\circ}$, complying well with the simulated results. The angular absorption performance for $\mathrm{TE}$ incidences, however, seems to be less efficient at $\theta=60^{\circ}$, simultaneously with a decrease of absorption bandwidth, which is more obvious than that of simulated results. Considering the imperfects in fabrication and the resistor assembly, the measured results are in rough agreement with simulated results. The measured results have demonstrated the efficient broadband absorption by the proposed metamaterial absorber for TM- and TE-polarized incidence with different incident angles. With proper optimization, it may achieve even broader microwave absorption by introducing, for example, metasurface ground plane to excite multiple resonances in a continuous working band [14,15].

The proposed broadband metamaterial absorber is compared with some representative previous absorbers in terms of bandwidth, thickness, and polarization sensitivity. The relative bandwidth $(-10 \mathrm{~dB}$, or $90 \%$ absorption $)$ of an absorber is defined with respect to its center working frequency, while the relative thickness is defined as the ratio of total thickness to the lowest working wavelength. As shown in Table 1, the proposed metamaterial absorber has a relative bandwidth approaching to $80 \%$ within a total thickness of $0.066 \lambda$, working for both $x$ - and $y$-polarizations. Besides, the proposed absorber can provide a robust angular performance for large incident angle.

\section{Conclusion}

In conclusion, a high-performance thin-thickness, polarization-insensitive, and broadband microwave metamaterial absorber loaded with lumped elements has been designed, simulated, and experimentally verified. The working band of the proposed metamaterial absorber can cover the whole $\mathrm{X}$ - and $\mathrm{Ku}$-bands, with relatively stable angular performance for incident angle up to about $60^{\circ}$. The design principle of the proposed metamaterial absorber is dependent on the loading of lossy materials, which can be potentially extended to other frequency band or even scaled at much high frequencies such as millimeter wave band and terahertz. In addition, our proposal may find potential uses in military applications such as electromagnetic compatibility, target cloaking, etc.

This work is partially supported by National Key Research and Development Program of China (Grant No. 2017YFA0700201), the National Nature Science Foundation of China (61801207, 61731010, 61671231, 61571218), project funded by China Postdoctoral Science Foundation (2017M620202), and partially supported by Fundamental Research Funds for the Central Universities, PAPD of Jiangsu Higher Education Institutions, and Jiangsu Key Laboratory of Advanced Techniques for Manipulating Electromagnetic Waves.

\section{References}

1. G.T. Ruck, D.E. Barrick, W.D. Stuart, C.K. Krichbaum, Radar cross section handbook (Plenum Press, New York, 1970)

2. Y. Ra'di, C.R. Simovski, S.A. Tretyakov, Thin perfect absorbers for electromagnetic waves: theory, design, and realizations, Phys. Rev. Appl. 3, 37001 (2015)

3. C.M. Watts, X. Liu, W.J. Padilla, Metamaterial electromagnetic wave absorbers, Adv. Mater. 24, OP98 (2012)

4. N.I. Landy, C.M. Bingham, T. Tyler, N. Jokerst, D.R. Smith, W.J. Padilla, Design, theory, and measurement of a polarization-insensitive absorber for terahertz imaging, Phys. Rev. B 79, 125104 (2009)

5. B. Zhu, Y. Feng, J. Zhao, C. Huang, T. Jiang, Switchable metamaterial reflector/absorber for different polarized electromagnetic waves, Appl. Phys. Lett. 97, 51906 (2010)

6. D. Shrekenhamer, W.C. Chen, W.J. Padilla, Liquid crystal tunable metamaterial absorber, Phys. Rev. Lett. 110, 177403 (2013) 
7. K. Chen, N. Jia, B. Sima, B. Zhu, J. Zhao, Y. Feng, T. Jiang, Microwave absorber based on permeability-near-zero metamaterial made of Swiss roll structures, J. Phys. D 48, 455304 (2015)

8. M. Diem, T. Koschny, C.M. Soukoulis, Wide-angle perfect absorber/thermal emitter in the terahertz regime, Phys. Rev. B 79, 033101 (2009)

9. S. Liu, H. Chen, T.J. Cui, A broadband terahertz absorber using multi-layer stacked bars, Appl. Phys. Lett. 106, 151601 (2015)

10. Y. Cui, Y. He, Y. Jin, F. Ding, L. Yang, Y. Ye, S. Zhong, Y. Lin, S. He, Plasmonic and metamaterial structures as electromagnetic absorbers, Laser Photon. Rev. 8, 495 (2014)

11. H. Tao, C.M. Bingham, D. Pilon, K. Fan, A.C. Strikwerda, D. Shrekenhamer, W.J. Padilla, X. Zhang, R.D. Averitt, A dual band terahertz metamaterial absorber, J. Phys. D 43 , $225102(2010)$

12. H.-X. Xu, G.-M. Wang, M.-Q. Qi, J.-G. Liang, J.-Q. Gong, Z.-M. Xu, Triple-band polarization-insensitive wide-angle ultra-miniature metamaterial transmission line absorber, Phys. Rev. B 86, 205104 (2012)

13. W.W. Salisbury, Absorbent body for electromagnetic waves, U.S. Patent No. 2,599,944 (1952)

14. Z. Zhou, K. Chen, B. Zhu, J. Zhao, Y. Feng, Y. Li, Ultrawideband microwave absorption by design and optimization of metasurface Salisbury screen, IEEE Access 6, 26843 (2018)

15. Z. Zhou, K. Chen, J. Zhao, P. Chen, T. Jiang, B. Zhu, Y. Feng, Y. Li, Metasurface Salisbury screen: achieving ultra-wideband microwave absorption, Opt. Express 25, 30241 (2017)

16. Y. Cheng, Y. Nie, X. Wang, R. Gong, Adjustable low frequency and broadband metamaterial absorber based on magnetic rubber plate and cross resonator, J. Appl. Phys. 115, $64902(2014)$

17. W. Li, T. Wu, W. Wang, J. Guan, P. Zhai, Integrating nonplanar metamaterials with magnetic absorbing materials to yield ultra-broadband microwave hybrid absorbers, Appl. Phys. Lett. 104, 22903 (2014)
18. B. Kearney, F. Alves, D. Grbovic, G. Karunasiri, Terahertz metamaterial absorbers with an embedded resistive layer, Opt. Mater. Express 3, 1020 (2013)

19. F. Costa, A. Monorchio, G. Manara, Analysis and design of ultra thin electromagnetic absorbers comprising resistively loaded high impedance surfaces, IEEE Trans. Antennas Propag. 58, 1551 (2010)

20. Y.Z. Cheng, R.Z. Gong, Y. Nie, X. Wang, A wideband metamaterial absorber based on a magnetic resonator loaded with lumped resistors, Chin. Phys. B 21, 127801 (2012)

21. Y.Z. Cheng, Y. Wang, Y. Nie, R. Zhou Gong, X. Xiong, $\mathrm{X}$. Wang, Design, fabrication and measurement of a broadband polarization-insensitive metamaterial absorber based on lumped elements, J. Appl. Phys. 111, 44902 (2012)

22. D. Ye, Z. Wang, K. Xu, H. Li, J. Huangfu, Z. Wang, L. Ran, Ultrawideband dispersion control of a metamaterial surface for perfectly-matched-layer-like absorption, Phys. Rev. Lett. 111, 187402 (2013)

23. N.V. Dung, P.V. Tuong, Y.J. Yoo, Y.J. Kim, B.S. Tung, V.D. Lam, J.Y. Rhee, K.W. Kim, Y.H. Kim, L.Y. Chen, Perfect and broad absorption by the active control of electric resonance in metamaterial, J. Opt. 17, 45105 (2015)

24. N.I. Landy, S. Sajuyigbe, J.J. Mock, D.R. Smith, W.J. Padilla, Perfect metamaterial absorber, Phys. Rev. Lett. 100, 207402 (2008)

25. Y. Shen, J. Zhang, Y. Pang, L. Zheng, J. Wang, H. Ma, S. Qu, Thermally tunable ultra-wideband metamaterial absorbers based on three-dimensional water-substrate construction, Sci. Rep. 8, 4423 (2018)

26. Y. Shen, J. Zhang, Y. Pang, J. Wang, H. Ma, S. Qu, Transparent broadband metamaterial absorber enhanced by water-substrate incorporation, Opt. Express 26, 15665 (2018)

27. P.-Y. Chen, C. Argyropoulos, A. Alù, Broadening the cloaking bandwidth with non-Foster metasurfaces, Phys. Rev. Lett. 111, 233001 (2013)

28. D.M. Pozar, Microwave engineering (John Wiley \& Sons, NJ, 2009)

Cite this article as: Ke Chen, Xinyao Luo, Guowen Ding, Junming Zhao, Yijun Feng, Tian Jiang, Broadband microwave metamaterial absorber with lumped resistor loading, EPJ Appl. Metamat. 6, 1 (2019) 